Präv Gesundheitsf 2009 · 4:225-226 DOI 10.1007/s11553-009-0208-7

Online publiziert: 6. November 2009

(c) Springer-Verlag 2009

\title{
J. Schmitt
}

Allergologie, Epidemiologie und Versorgungsforschung, Klinik und Poliklinik für

Dermatologie, Universitätsklinikum Carl Gustav Carus, Technische Universität Dresden

\section{Determinanten aktiver Gesundheitsförderung}

Wesentliche Voraussetzungen und Determinanten für eine aktive Gesundheitsförderung sind 1. der Zugang zu und die Qualität von Informationen zu modifizierbaren Einflussfaktoren von Gesundheit und Krankheit, 2. Determinanten der Umsetzung dieser Gesundheitsinformation in eine Modifikation des individuellen Gesundheitsverhaltens sowie 3. gesellschaftliche/interindividuelle Unterstützungsfaktoren zur Aufrechterhaltung des Gesundheitsverhaltens.

Im vorliegenden Heft werden diese Kernprinzipien der Prävention und Gesundheitsförderung bezogen auf unterschiedliche Szenarien beispielhaft analysiert.

Die ersten beiden Artikel dieses Heftes befassen sich mit dem Themenkomplex Gesundheitsinformation und biomedizinisches Wissen. Honekamp et al. evaluierten eine zukunftsweisende und vielversprechende bedarfsgerechte Informationsdarbietung im Internet am Beispiel Kopfschmerz. Die Autoren beschreiben das Potential und die Herausforderung von diagnosebezogenen Gesundheitsinformationen, einer Thematik, die in $\mathrm{Zu}$ kunft von großer Bedeutung sein könnte.

Impfen ist wie keine andere Thematik im Bereich Präventivmedizin Gegenstand kontroverser gesellschaftlicher und wissenschaftlicher Diskussionen. Aufklärung und Informationen finden auf verschiedenen Ebenen, jedoch meist auf die primären Zielgruppen wie z. B. junge Eltern ausgerichtet statt. Zeyer u. Knierim gelingt es mit ihrem Beitrag eine wichtige Lücke in diesem Themenkomplex zu schließen und die Leserschaft auf ein interessantes neues Feld der Impfprävention aufmerksam zu machen. Zielpopula- tion der Studie zur Wissensvermittlung über Nutzen und Risiko von Impfungen waren nicht Eltern oder ältere Menschen, sondern Studenten. Bei diesen jungen Erwachsenen wurde durch eine zielgruppenadäquate Aufklärung eine gesteigerte Gesundheitskompetenz erreicht, so dass eine Multiplikatorwirkung oder Mittlerfunktion im gesellschaftlichen Diskurs möglich scheint.

Einen weiteren Schwerpunkt des vorliegenden Heftes bilden Arbeiten zu Determinanten der Umsetzung von $\mathrm{Ge}$ sundheitsinformationen im Alltag u. a. bezogen auf körperliche Aktivität, sexuell übertragbare Erkrankungen und Kindergesundheit. Körperliche Inaktivität ist ein führender Risikofaktor für Morbidität und Mortalität. Oertel et al. erörtern in leserfreundlicher Weise die verschiedenen Möglichkeiten körperlicher Betätigung und gehen dabei auf das Nutzen-RisikoVerhältnis verschiedener Sportarten und Trainingsmethoden ein.

In einer repräsentativen Untersuchung an rund 10.000 Jugendlichen und Erwachsenen identifizieren Rütten et al. Barrieren der körperlichen Aktivität. Die Ergebnisse dieser Studie sind von großer praktischer Relevanz für das Design effektiver Maßnahmen der Gesundheitsförderung, weil sie modifizierbare Determinanten spezifisch für demographisch charakterisierte Subpopulationen herausstellen.

Kliche et al. berichten wichtige Ergebnisse einer Delphi-Konsensusstudie zum Stellenwert von Qualitätskriterien für Maßnahmen der Prävention und Gesundheitsförderung. Wesentlicher Vorteil dieser Konsensusmethode ist die Vermeidung der Dominanz einzelner Mitglieder 
des Expertenpanels, so dass eine generalisierbare Aussage erzielt werden kann.

Eine Vollerhebung des Rauchverhaltens und assoziierter Einflussgrößen bei Fünftklässlern von Thyrian et al. bestätigt den Zusammenhang von rauchendem Umfeld und eigenem Rauchverhalten der Kinder. Wichtiges Ergebnis ist darüber hinaus die Einteilung der Nichtraucher in Nieraucher und Probierer, was in Zukunft ein gezieltes „Targeting“ potenzieller jugendlicher Raucher vor der Einstiegsphase ermöglichen kann.

In vielen Bundesländern wird eine bessere Überwachung sowie Erweiterung der Kindervorsorgeuntersuchungen diskutiert. Stich et al. zeigen wesentliche Determinanten wie Nationalität, Muttersprache sowie Indikatoren der sozioökonomischen Position des Teilnahmeverhaltens an den U-Untersuchungen auf und können so helfen, bei möglicherweise bevorstehenden gesundheitspolitischen Maßnahmen eine hohe Effizienz zu erreichen, und so weiterhin zu einer evidenzbasierten Gesundheitspolitik beitragen.

Da HIV/AIDS in Osteuropa relativ hohe Prävalenzen und Inzidenzen hat, ist die Studie von Kouznetsov et al. über das Wissensniveau bei Aussiedlern aus der ehemaligen Sowjetunion gesellschaftlich hochrelevant. Aussiedler werden wie dargestellt von den laufenden HIV-Präventionsmaßnahmen in Deutschland nur ungenügend erreicht.

Linder u. Mengering berichten von einem Pilotprojekt mit Lebenskompetenztraining zur HIV/AIDS-Prävention sozial benachteiligter Kinder in der Republik Südafrika. Leider hat sich in dieser Untersuchung ein zunächst erzielter Wissensgewinn über die Infektionskrankheit als nicht nachhaltig erwiesen.

Die Beiträge von Beck und von Nolte beschreiben schließlich gesellschaftliche Faktoren für das Gesundheitsverhalten und gesundheitsrelevante „Outcomes“ bei Beschäftigten verschiedener Branchen. Beck u. Lenhardt präsentieren Ergebnisse einer Erwerbstätigenbefragung und zeigen auf, dass die Betriebsgröße für die Arbeitsbedingungen und -zufriedenheit als wesentliche Determinanten der Beschäftigtengesundheit durchaus eine Rolle spielt. Psychische Belastungen finden sich häufiger in größeren Unternehmen,

während Beschäftigte kleinerer Betriebe häufiger körperlichen Beanspruchungen ausgesetzt sind. Diese Studie bietet somit ebenfalls Anhaltspunkte für eine zielgerichtete betriebliche Prävention. Die Vermutung, dass eine übermäßige Verausgabungsbereitschaft von Bankern nicht gesundheitszuträglich ist, wird erstmals von Nolte bestätigt. Der Autor beschreibt die negativen Folgen von „Overcommitment“ auf die physische und psychische Gesundheit der Beschäftigten.

Abgerundet wird das Heft durch einen aufschlussreichen Überblick über Priorisierung und Unterstützung von PublicHealth-Forschung in Nord-, Süd- und Osteuropa. Der Artikel von Mannocci et al. verdeutlicht nicht nur die unterschiedlichen nationalen Forschungsprioritäten in Europa, sondern stellt auch eine von vielen Befragten angegebene vergleichsweise niedrige Priorität internationaler Public-Health-Forschung heraus und diskutiert mögliche Ursachen und Lösungsansätze.

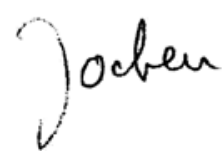

J. Schmitt

\section{Korrespondenzadresse \\ Dr. med. habil. J. Schmitt}

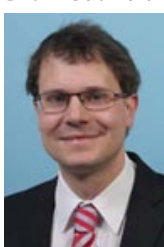

Allergologie, Epidemiologie

und Versorgungsforschung,

Klinik und Poliklinik für Dermatologie, Universitätsklinikum Carl Gustav Carus, Technische Universität, Fetscherstraße 74, 01307 Dresden jochen.schmitt@ uniklinikum-dresden.de 